\title{
Clinical Functional MRI of the kidneys
}

\author{
Shila Pazahr $\cdot$ Andreas Boss $\cdot$ Cristina Rossi
}

Published online: 21 March 2013

(c) Springer Science+Business Media New York 2013

\begin{abstract}
In functional renal magnetic resonance imaging (MRI), advanced techniques are applied to obtain information on a functional and molecular level from the kidney tissue beyond pure morphology. Techniques such as diffusion-weighted and diffusion tensor imaging, arterial spin labelling, and blood oxygenation level-dependent imaging provide potential biomarkers of organ function. Moreover, dynamic contrast-enhanced techniques after the intravenous injection of gadolinium-chelates may be used to assess glomerular filtration and urinary excretion. This review summarizes recent developments of contrast- and non-contrast-enhanced MRI techniques for assessment of renal function in a clinical setting. The physiological background and the sequence techniques are described in detail. Potential clinical applications of the different techniques are discussed regarding their potential usefulness in the assessment of parenchymal diseases, urinary tract anomalies, transplant kidney function, and renal masses.
\end{abstract}

Keywords MRI - Kidney - DWI - DTI - ASL - BOLD · Renal function

\section{Introduction}

Besides allowing excellent morphological visualization of soft tissue, magnetic resonance imaging (MRI) can provide

S. Pazahr $(\bowtie) \cdot$ A. Boss $\cdot$ C. Rossi

Department of Diagnostic and Interventional Radiology,

University Hospital Zurich, Rämistr. 100, 8091 Zurich,

Switzerland

e-mail: shila.pazahr@usz.ch

A. Boss

e-mail: Andreas.Boss@usz.ch additional information, on a cellular and molecular level, for assessing functional aspects of organs. Though initially developed for neuroimaging, several advanced MRI techniques have recently been adapted for kidney evaluation. The restriction of the free water diffusion, which can be quantified using diffusion-weighted imaging (DWI) techniques, has been reported in patients affected by parenchymal kidney diseases. Moreover, the water diffusivity measurements can be applied for differentiation of renal masses. In the renal pyramids, anisotropy of water diffusion, which can be visualized using diffusion tensor imaging (DTI), reflects the directionality of the tissue. Renal tissue perfusion may be measured without application of contrast-media using arterial spin labelling (ASL) techniques. Moreover, renal tissue oxygenation can be assessed non-invasively with blood-oxygenation leveldependent (BOLD) MRI, which is interesting because of the physiological hypoxia in the renal medulla. Measuring the renal uptake of gadolinium-based contrast-media after bolus injection allows for computation of the differential renal function similar to techniques of nuclear scintigraphy. Moreover, by monitoring of the excretion of the contrastmedia into the renal pelvis and ureter, obstacles to the urinary drainage can be detected and assessed (Table 1).

Application of these advanced MRI techniques in the abdomen requires several optimization steps accounting for motion and strong static magnetic field inhomogeneities. A first issue consists in the prevention of motion artifacts. As an alternative to breath-hold acquisition, organ movement due to respiration can be compensated by navigator or breathing-belt triggering [1]. The different tissue susceptibilities result in magnetic field inhomogeneities, which may severely undermine image quality in the abdomen. In the brain, DWI, DTI, BOLD, and ASL sequences use an echo-planar imaging (EPI) acquisition technique for 
Table 1 Current publications on functional MR of the kidney

\begin{tabular}{|c|c|c|c|c|c|}
\hline Methods & $\begin{array}{l}\text { Assessment } \\
\text { of transplanted } \\
\text { kidney }\end{array}$ & $\begin{array}{l}\text { Assessment } \\
\text { of renal } \\
\text { masses }\end{array}$ & $\begin{array}{l}\text { Assessment } \\
\text { of diffuse } \\
\text { kidney disease }\end{array}$ & $\begin{array}{l}\text { Assessment of } \\
\text { reproducibility (healthy } \\
\text { volunteers) }\end{array}$ & Animal study \\
\hline $\begin{array}{r}\text { DWI/ } \\
\text { DTI }\end{array}$ & $\begin{array}{l}\text { Palmucci et al. [19] } \\
\text { Thoeny and De Keyzer }[17 \bullet \bullet] \\
\text { Hueper et al. [18] }\end{array}$ & $\begin{array}{l}\text { Yu et al. [15] } \\
\text { Inci et al. [14] } \\
\text { Chandarana et al. [16] } \\
\text { Zhang et al. [13] }\end{array}$ & Lu et al. [20] & $\begin{array}{l}\text { Sigmund et al. [11] } \\
\text { Wittsack et al. [12] } \\
\text { Rosenkrantz et al. [54] }\end{array}$ & Hueper et al. [21] \\
\hline ASL & Artz et al. [26] & $\begin{array}{l}\text { Pedrosa et al. [28] } \\
\text { Lanzman et al. [29] }\end{array}$ & Rossi et al. [27] & $\begin{array}{l}\text { Cutajar et al. [23] } \\
\text { Wu et al. [24] } \\
\text { Boss et al. [22] }\end{array}$ & Schor-Bardach et al. [30] \\
\hline BOLD & $\begin{array}{l}\text { Park et al. [37] } \\
\text { Xiao et al. [36] }\end{array}$ & Min et al. [38] & $\begin{array}{l}\text { Yin et al. [34] } \\
\text { Michaely et al. [43] } \\
\text { Xin-Long et al. [33] }\end{array}$ & $\begin{array}{l}\text { Park et al. [37] } \\
\text { Donati et al. [42] } \\
\text { Simon-Zoula et al. [32] }\end{array}$ & $\begin{array}{l}\text { Zhang et al. [40••] } \\
\text { Haneder et al. [41] } \\
\text { Warner et al. [39] } \\
\text { Prasad et al. [35•] } \\
\text { Pedersen et al. [31] }\end{array}$ \\
\hline
\end{tabular}

$D W I$ diffusion-weighted imaging, $D T I$ diffusion tensor imaging, $A S L$ arterial spin labeling, $B O L D$ blood oxygen level dependent

single-shot image acquisition, which is more susceptible to image distortions and signal dephasing caused by magnetic field inhomogeneities compared to conventional image readout strategies. These problems can now in part be compensated by dedicated sequence techniques, powerful gradient systems and parallel imaging methods [2•, 3, 4].

Most of the functional imaging techniques suffer from low signal yield. Therefore, measurement at higher field strength of $3 \mathrm{~T}$ seems advantageous. However, dielectric effects caused by $B_{1}$ field interferences may result in local signal voids and mimic pathological lesions [5]. With radiofrequency (RF) shimming and parallel RF transmission, techniques have been developed within the last years providing more homogeneous flip angle distributions within the abdomen [6].

Several of the described functional imaging techniques might be especially interesting for application in patients with renal insufficiency, which are known to be at risk to develop nephrogenic systemic fibrosis after administration of gadolinium-based contrast agents [7], as those techniques provide functional information without injection of contrast-media.

The aim of this review article is to provide an overview on techniques of DWI, DTI, ASL, BOLD MRI, and contrastenhanced MR nephrography, as well as the most recent developments.

\section{DWI and DTI}

Technique

As during their random motion water molecules experience the microscopic architecture of the tissue, DWI is often applied to provide anatomical information on a microscopic scale. In DW sequences, diffusion sensitizing gradients are applied after spin excitation resulting in signal attenuation in dependence on the degree of diffusion and the so-called " $b$ value" (in units of $\mathrm{s} / \mathrm{mm}^{2}$ ). The $b$ value is a sequence parameter that can be computed from the timing and strength of the applied diffusion-encoding gradients. Pure diffusion occurring in an isotropic and homogeneous tissue results in a mono-exponential MR signal decay for increasing $b$ values. Multiple $b$ value measurements allow quantitative assessment of the apparent diffusion coefficient (ADC) by fitting of the MR signal to the expression:

$S(b)=S(0) \cdot \mathrm{e}^{-b \cdot \mathrm{ADC}}$.

Typically, at least two $b$ values are acquired: A measurement with $b=0 \mathrm{~s} / \mathrm{mm}^{2}$ (" $b_{0}$ ") can be used as reference and a higher $b$ value is applied in a second measurement. If only two $b$ values are used, Eq. 1 can be solved for the ADC as:

$\mathrm{ADC}=\frac{\log \frac{S(0)}{S(b)}}{b}$.

In tissues this approach is only suitable for diffusion measurements, if "high" $b$ values (i.e., $>200 \mathrm{~s} / \mathrm{mm}^{2}$ ) are applied. The reason is that blood flow in randomly distributed capillaries mimics a pseudo-diffusion effect, which dominates signal attenuation for low $b$ values [8]. The expression intra-voxel incoherent motion (IVIM) has been coined to indicate spins moving in fluids due to capillary perfusion and molecular diffusion. When the $b$ value ranges between 0 and $1,000 \mathrm{~s} / \mathrm{mm}^{2}$, the signal intensity curve measured in the corresponding MR images 
presents a bi-exponential pattern, mainly reflecting tissue perfusion for $b<100 \mathrm{~s} / \mathrm{mm}^{2}$ and water diffusion in tissue for $b>200 \mathrm{~s} / \mathrm{mm}^{2}$. The signal behaviour of IVIM can be described by the following equation:

$$
S(b)=S(0) \cdot\left(f_{\mathrm{p}} \cdot \mathrm{e}^{-b D_{\mathrm{f}}}+\left(1-f_{\mathrm{p}}\right) \cdot \mathrm{e}^{-b \mathrm{ADC}}\right),
$$

with $f_{\mathrm{p}}$ meaning the perfusion fraction and $D_{\mathrm{f}}$ describing the perfusion related signal attenuation (Fig. 1). Typically, the equation is evaluated in two steps. First, the higher $b$ values are applied in a mono-exponential fit assuming that the contribution of the perfusion to the signal intensity at high $b$ values is zero. In a second step, the computed value for $\mathrm{ADC}$ is kept constant and $S(0), f_{\mathrm{p}}$ and $D_{\mathrm{f}}$ are variables of a second, bi-exponential fit.

Mainly due to the presence of collecting ducts and vessels radially oriented towards the renal papillae, molecular diffusion in kidneys presents direction-dependent properties $[9,10]$. For this reason the computation of a diffusion tensor, rather than the estimation of a single ADC value, provides a more comprehensive characterization of water diffusion in kidneys (Fig. 2).

\section{Applications}

Diffusion parameters may indirectly indicate renal dysfunction related to pathological alteration of tissue microarchitecture (e.g., atrophy or sclerosis). Moreover, signal changes in DW images acquired using low $b$ values may reveal renal dysfunction associated to changes in capillary perfusion.

Good reproducibility of the diffusion parameters (average coefficient of variation [CV] of $3.7 \%$ for cortex and of $5.0 \%$ for the medulla) was reported by Sigmund et al. [11]. Wittsack et al. [12] reported a dependence of ADC and $f_{\mathrm{p}}$ values from the phase of the cardiac cycle proving the influence of the pulsatile blood flow on the evaluation of the parameters in kidneys.

In a study conducted in patients with renal lesions at 1.5 $\mathrm{T}$, Zhang et al. [13] reported ADC values estimated by mono-exponential fitting of the MR signal of images acquired with high $b$ values $\left(0-500-1,000 \mathrm{~s} / \mathrm{mm}^{2}\right)$. Solid enhancing lesions presented significantly lower ADC values as compared to benign cysts and non-enhancing lesions. Inci et al. [14] recently showed that DWI allows distinguishing cystic renal lesions with Bosniak category I from II to III, which may influence patient treatment. Inci and colleagues also suggested that ADC values may help differentiating renal cell carcinoma (RCC) subtypes (papillary RCC exhibits low ADC values, clear-cell RCC intermediate and chromophobe RCC relatively higher $\mathrm{ADC}$ values). Yu et al. [15] recently reported the feasibility of ADC measurements performed at $3 \mathrm{~T}$ for differentiation and classification of RCC. In the mentioned studies, a mono-exponential model was applied for ADC computation. Applying the bi-exponential IVIM model, Chandarana et al. [16] found significantly higher perfusion fraction and lower diffusion in solid enhancing lesions as compared to non-enhancing lesions.

Recently, few studies investigated the possibility of detecting organ dysfunction in transplanted kidneys using DW-MRI techniques [17••, 18]. Palmucci et al. [19] reported significantly higher ADC values of the renal cortex in transplanted kidneys with a normal glomerular filtration rate, GFR ( $>60 \mathrm{ml} / \mathrm{min})$ as compared with functionally restricted grafts (GFR $\leq 30 \mathrm{ml} / \mathrm{min}$ ). The DTI parameters (such as fractional anisotropy, FA) were computed in renal allografts and compared to the results obtained in healthy volunteers [18]. Significantly lower $\mathrm{ADC}$ and FA values were found in allografts as compared to control subjects. Moreover, in the cohort of patients with renal transplants, the mean FA correlated to the estimated GFR (eGFR), suggesting that DTI may help detecting allograft dysfunction [18].

Lu et al. [20] reported reduced medullary FA in diabetic patients with and without impaired renal function compared with healthy volunteers, suggesting that DTI could be helpful for early diagnosis of diabetic nephropathy. Furthermore, the results reported by Hueper et al. [21] in a rat model of diabetic nephropathy suggest that a strong restriction of water diffusion in renal tissue may reflect glomerulosclerosis, interstitial fibrosis, and tubular damage.
Fig. 1 An image without diffusion weighting $\left(b=0 \mathrm{~s} / \mathrm{mm}^{2}\right)$ is displayed indicating an ROI defining the renal cortex. The graph on the right side shows signal intensities from the ROI in diffusion sensitive measurements using multiple $b$ values $(0,10,20,60,150,300$, $\left.500,1000 \mathrm{~s} / \mathrm{mm}^{2}\right)$, and a corresponding IVIM fit
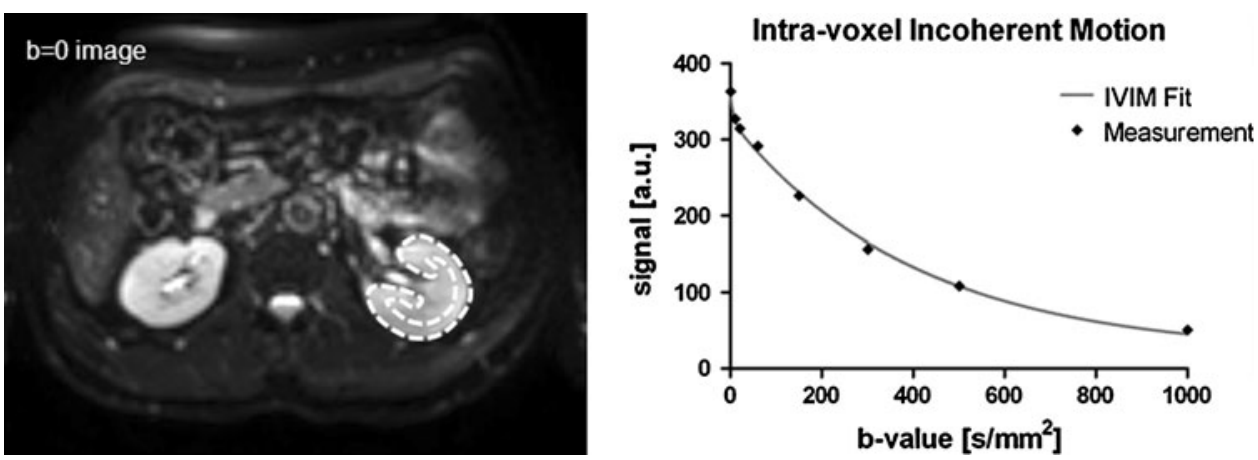


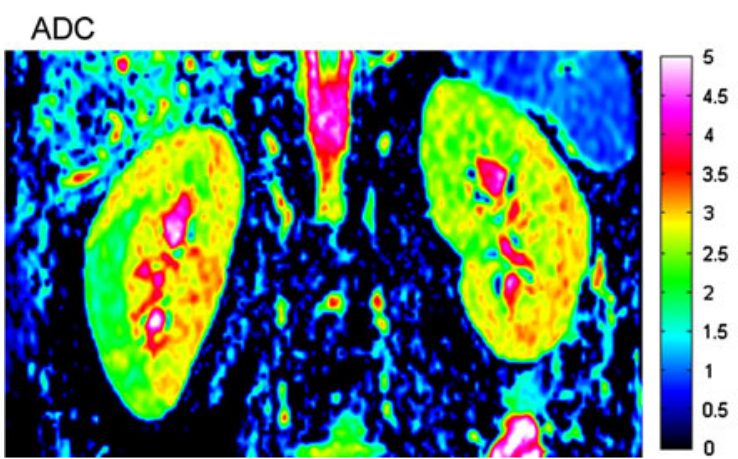

FA

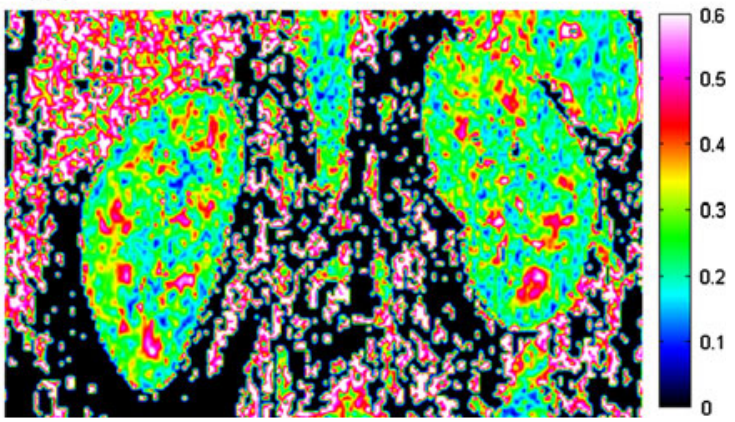

Fig. 2 Results from diffusion tensor images in coronary orientation. The ADC map is displayed on the left side $\left(\mathrm{scale}\right.$ in $\left.10^{-3} \mathrm{~mm} / \mathrm{s}\right)$, the corresponding FA map on the right side. Strongest diffusion anisotropy can be seen in the renal medulla

\section{Arterial Spin Labeling}

Technique

The ASL is a non-invasive and -contrast-enhanced technique for visualization or even quantification of tissue perfusion. In ASL RF-labelled blood water is used as endogenous tracer. Many different labelling techniques have meanwhile been developed. In principle, two methods of ASL can be applied (i.e., continuous ASL [cASL] and pulsed ASL [pASL]) which differ in the way the magnetization of blood water is labelled: In cASL, the blood water is continuously labelled in a supplying artery, e.g., with a separate labelling coil. Image readout is done under steady-state conditions. This technique has the advantage of providing relatively high signal yield. Disadvantages are the need for dedicated hardware, the high RF energy deposition, and the need to consider transit time effects appropriately, which are related to the (in principle unknown) traveling time of the labelled blood from the labelling slab to the imaging plane. On the other hand, the idea behind so-called pASL is to perform the blood water labelling by an RF pre-pulse transmitted by the regular coil. This RF pre-pulse has to be applied at a distinct time prior to recording data for imaging. One of the most commonly applied techniques is the flow-sensitive alternating inversion recovery (FAIR) spin preparation, which uses an inversion pulse for spin labeling. Its principle is illustrated in Fig. 3. In this technique, two acquisitions with different labelling are alternatingly applied: one acquisition with selective inversion of a slab that is slightly larger than the imaging slice (no labelling of in-flowing blood) and one image with global inversion of all blood within the imaging region. Image acquisition typically takes place about $1.2 \mathrm{~s}$ after the inversion pulse. Subtraction of the image with global inversion from the image with selective inversion results in a perfusion-weighted image, as the difference between the two images is caused by the non-labelled blood moving from outside the inversion slab of the image with selective inversion into the imaging plane. Quantitative perfusion values in $\mathrm{ml} / 100 \mathrm{~g}$ tissue/min can be computed from the following simplified solution of the extended Bloch equations:

$f=\frac{\lambda}{2 \cdot \mathrm{TI}} \frac{\Delta M(\mathrm{TI})}{M_{0}} \exp \left(\frac{\mathrm{TI}}{T_{1}}\right)$

where $f$ denotes the perfusion, $\lambda$ the tissue/blood partition coefficient (typically set to 0.9 in kidneys), $\Delta M$ (TI) the difference of magnetization at a time TI after slice-selective and global preparation, $M_{0}$ the equilibrium magnetization without application of a preparation pulse measured from a proton-density-weighted image without ASL preparation, and $T_{1}$ the tissue longitudinal relaxation time.

\section{Applications}

Feasibility and reproducibility of ASL measurements in kidneys have been investigated in numerous studies [3, 22-24]. The main challenge is related to the relatively low signal-to-noise ratio (SNR) of the ASL images due to the image subtraction, which requires averaging of a high number of acquisitions. Especially quantification of the low perfusion in renal medulla is still a challenge. Moreover, body motion between subsequent image acquisitions may strongly affect the accuracy of the perfusion measurements in abdominal organs [25].

Reproducibility and stability of a multi-TI pASL approach (3D GRASE) in free breathing was recently tested at $1.5 \mathrm{~T}$ in healthy volunteers [23]. Although renal perfusion is known to vary even over the course of a day in the same person, Cutajar et al. [23] reported a CV of the perfusion values of $7.52 \%$ for the whole kidney. Wu et al. [24] recently compared measurements of renal blood flow using ASL (pseudo-continuous labeling scheme) and dynamic contrast enhancement imaging at $3 \mathrm{~T}$. Acceptable agreement was found in the renal cortex, while inconsistent 
Fig. 3 Schematic diagram showing the principle of ASL. Subtraction of the image recorded after non-selective inversion from the image recorded after selective inversion results in a perfusionweighted image due to blood flowing from the differently labelled regions into the imaging plane. With knowledge of the equilibrium magnetization $M_{0}$ (as indicated by a proton-density-weighted image) and the $T_{1}$ longitudinal relaxation time, a quantitative perfusion map can be calculated using the extended Bloch equations

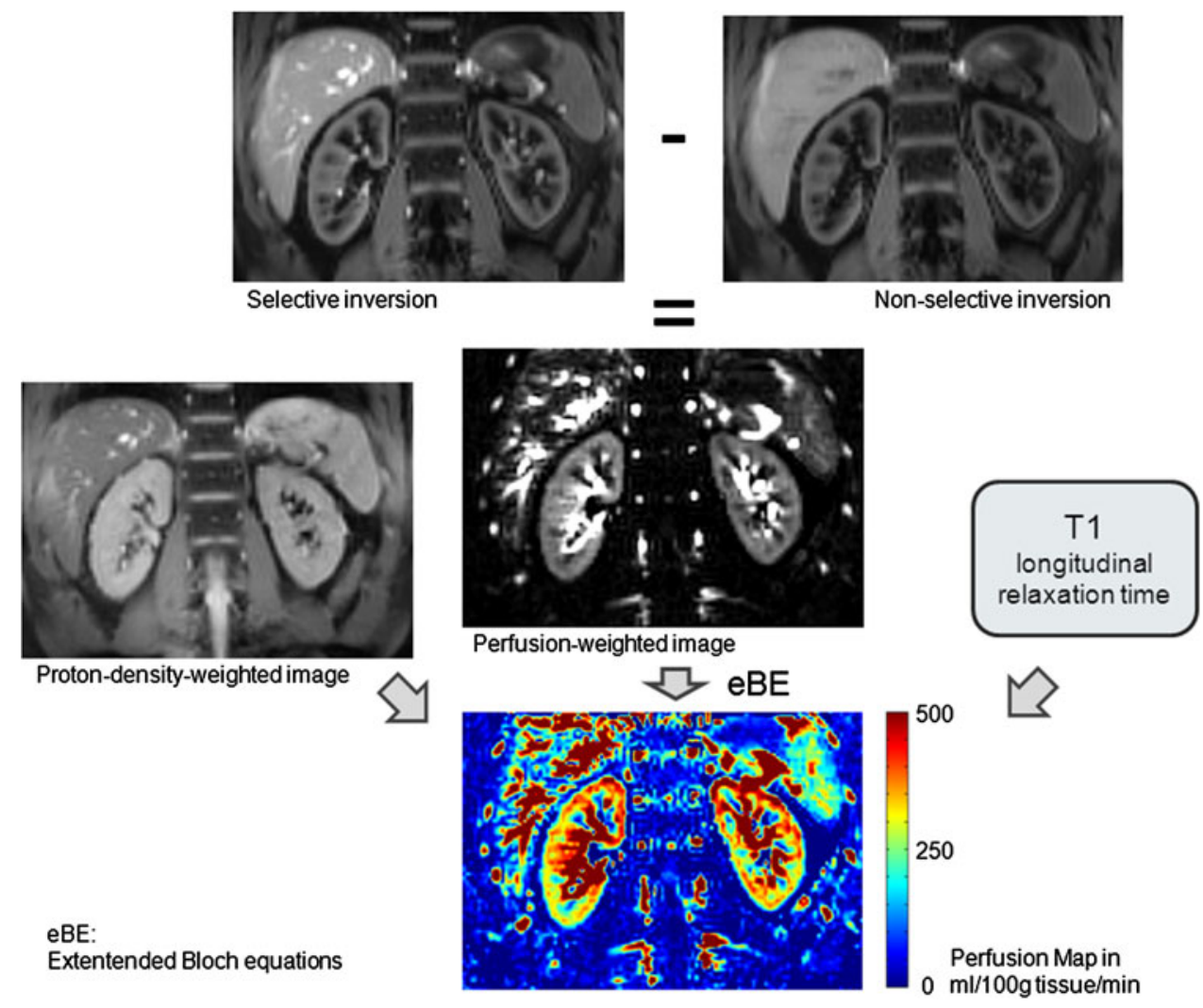

values were reported for the medulla. Artz et al. [26] investigated the reproducibility of ASL measurements using a respiratory-triggered FAIR TrueFISP sequence in native and transplanted kidneys. Again, reasonable reproducibility was found for the renal cortex (CV for measurements within the same day 4.8-6.0\% and $\mathrm{CV}$ of 7.6-13.1\% for measurements at different days), but perfusion measurements in the medulla showed poor reproducibility (CV 16.7-37\%).

Rossi et al. [27] performed a histogram analysis of ASL perfusion maps in patients with moderate chronic kidney disease. Significant differences in the perfusion metric of the renal cortex were found between healthy volunteers and the patients. These preliminary results suggest that renal cortical perfusion may be a marker of the onset of the renal dysfunction and could be applied for follow-up of the disease.

Pedrosa et al. [28] reported on ASL measurements for assessment of the vascularity of renal masses in a small cohort of patients with impaired renal function at $1.5 \mathrm{~T}$. More recently Lanzman et al. [29] have presented an ASL study indicating higher perfusion levels in oncocytomas than in RCCs, and reduced perfusion in papillary RCCs as compared to other RCC subtypes. Schor-Bardach et al. [30] showed in an animal model of RCC that ASL can provide information on tumor viability.

\section{BOLD MRI}

Technique

BOLD MRI uses the paramagnetic properties of deoxyhaemoglobin to indirectly gain information on the oxygen metabolism in kidneys [4]. Due to the different magnetic properties of oxy- and deoxyhaemoglobin, changes in the oxygenation state of blood (e.g., due to furosemide administration) result in variation of the intra-vascular magnetic susceptibility and, in turn, affect the contrast of $T_{2}^{*}$-weighted images in areas close to the blood vessels. A typical BOLD experiment consists of recording single echo $T_{2}^{*}$-weighted images $(1.5 \mathrm{~T}$, TE circa $50 \mathrm{~ms} ; 3 \mathrm{~T}$, TE circa $25 \mathrm{~ms}$ ) before and after induced changes in the oxygenation of the tissue. The signal difference between the two images allows qualitative mapping of areas of oxygen consumption. However, due to the dependence of the BOLD-signal change on the experimental set-up, this method does not allow absolute quantification of the oxygenation status of the tissue.

As an alternative, multi-echo gradient recalled echo (GRE) images can be acquired and the relaxivity $\left(R_{2}^{*}=1 / T_{2}^{*}\right)$ of the renal tissue can be assessed by fitting the MR signal to the expression (Fig. 4): 


$$
S(\mathrm{TE})=S(0) \cdot \mathrm{e}^{-R_{2}^{*} \cdot \mathrm{TE}}
$$

This approach is a non-invasive way to infer renal haemodynamic. However, for an inter-study comparison of relaxivity values, differences in the experimental set-up (e.g., shim quality, signal noise, movement compensation, image resolution) must be taken into account, since those parameters can considerably affect the computation of the $R_{2}^{*}$ value.

Although EPI sequences are typically used for the measurement of the BOLD effect in the brain, GRE techniques are usually used for renal BOLD MRI. These techniques are less prone to susceptibility artefacts that are prominent at the upper and lower pole of the left kidney because of the lung and the bowel gas.

\section{Applications}

The validity of the BOLD signal for monitoring of renal oxygenation has been investigated in animal studies: Renal BOLD MRI was demonstrated to reflect oxygen consumption in pigs by comparison with oxygen partial pressure $\left(p_{\mathrm{O}_{2}}\right)$ measurements performed with oxygen-sensitive microelectrodes [31].

At the baseline, $R_{2}^{*}$ values in the order of $12 \mathrm{~s}^{-1}$ and of $16 \mathrm{~s}^{-1}$ were reported at $1.5 \mathrm{~T}$ in the cortex and the medulla, respectively [32]. Numerous studies focused on the quantification of baseline renal relaxivity values under pathological conditions. Xin-Long et al. [33] reported significantly higher relaxivity values of both, cortex and medulla, in patients with chronic glomerulonephritis as compared to healthy volunteers. These results suggest possible anoxia in the medullary and cortical areas in patients. Yin et al. [34] recently applied BOLD MRI to monitor and assess renal hypoxia in diabetes. The authors reported a significant increase of both medullary and cortical $R_{2}^{*}$ values in diabetes compared with healthy volunteers. In this study a positive (negative) correlation between medullary (cortical) $R_{2}^{*}$ and eGFR was found. Similar findings were previously reported in a rat model of type 2 diabetes [35•]. Furthermore, a decrease of the medullar over cortical $R_{2}^{*}$ ratio with the progress of diabetic nephropathy was found [35•]. Xiao et al. [36] investigated at 1.5 T the value of BOLD MRI for the evaluation and detection of acute rejections after renal transplantation. Significant $R_{2}^{*}$ decrease in both, cortex and medulla was found in acute rejection allografts as compared with normal functioning allografts. In nine patients with acute rejection or acute tubular necrosis abnormal $R_{2}^{*}$ and decreased cortico-medullary demarcation were found. These results suggest that BOLD MRI may reflect pathophysiological changes related to increased risk of acute rejection and acute tubular necrosis. Comparable $R_{2}^{*}$ values were found in normal functioning renal grafts and in healthy volunteers. Park et al. [37] reported significantly lower $R_{2}^{*}$
Fig. $4 T_{2}^{*}$ relaxation times and $R_{2}^{*}$ relaxation rates can be computed for all picture elements from the data set of a multi-echo gradient-echo sequence. A mono-exponential fit was applied pixel-wise for calculation of the presented $T_{2}^{*}$ and $R_{2}^{*}$ maps
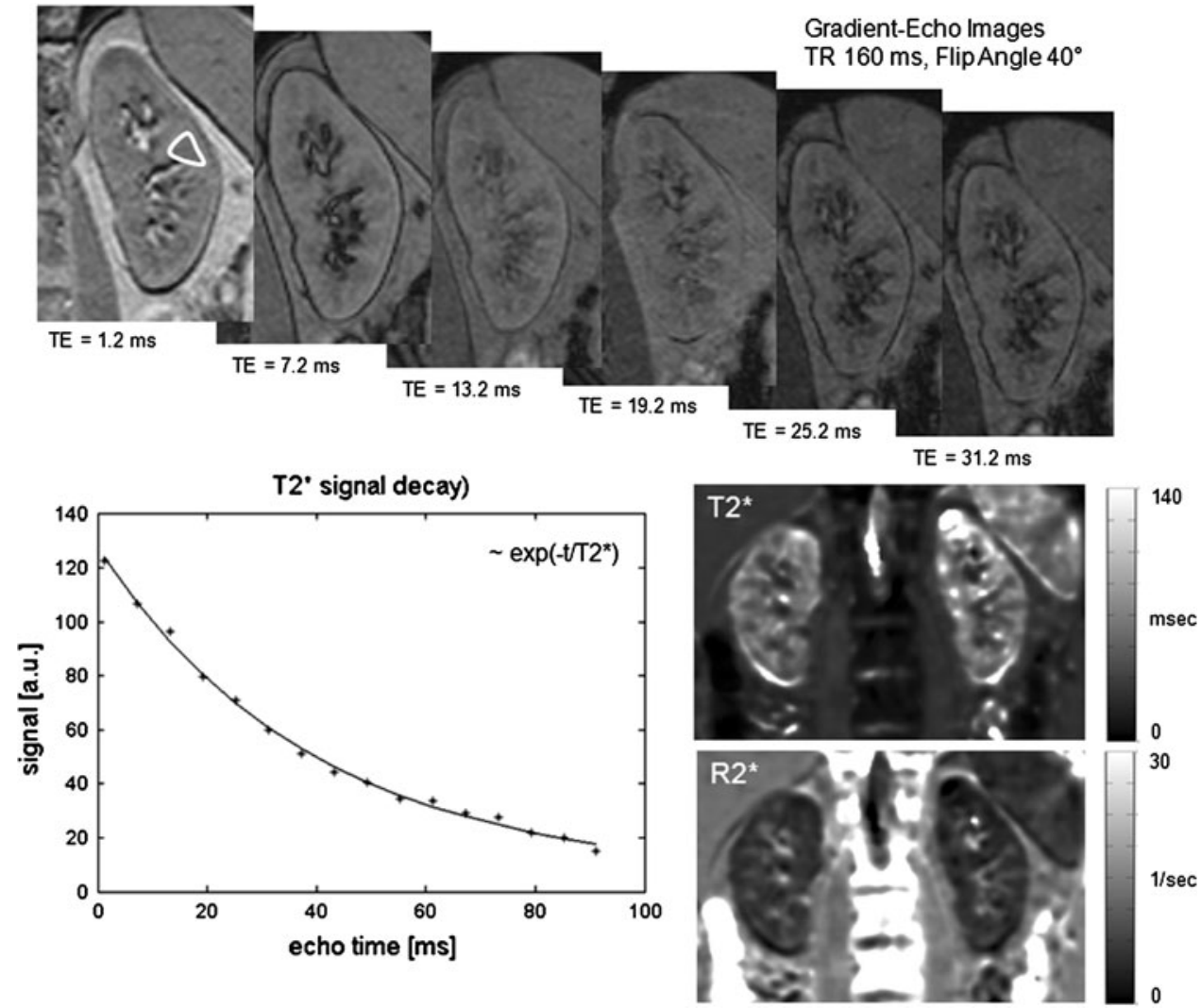
values over the medulla in acute allografts rejection at $3 \mathrm{~T}$. Min et al. [38] could show in a relatively large number (more than 100) of renal lesions that BOLD imaging is feasible at 3 T MR systems. Renal lesions were divided in four subgroups (RCC, angiomyolipoma, simple cysts and hemorrhagic cysts) and showed significantly different $R_{2}^{*}$ values [38].

Few studies focussed on the renal BOLD response to interventions. In an animal study with domestic pigs, Warner et al. [39] investigated the changes in tissue oxygenation as well as $R_{2}^{*}$ values measured with BOLD imaging at $1.5 \mathrm{~T}$ after administration of the loop diuretic furosemide and shortacting proximal diuretic. Medullary and cortical oxygenation induced by these selective diuretics was detectable by BOLD MRI. However, as cortical $R_{2}^{*}$ values changed after furosemide administration, the authors suggested that $R_{2}^{*}$ quantification may reflect, beside oxygenation, haemodynamic effects. In order to analyse the effects of ionated contrastmedia on renal hemodynamics and oxygenation, Zhang et al. [40••] injected iopamidol to white rabbits and acquired ASL and BOLD MR images at $3 \mathrm{~T}$. After the injection, prolonged hypoperfusion in the whole kidney tissue as well as decreased tissue oxygenation in the inner stripes of the outer medulla was found. After $72 \mathrm{~h}$, the measured renal blood flow and oxygenation corresponded to the baseline measured before iopamidol was injected. Haneder et al. [41] also investigated the changes induced by ionated contrast agent in kidney oxygenation in mini-pigs using BOLD imaging. In contrast to Zhang et al. [40••], $55 \mathrm{~min}$ after injection of iopromide and iodixanol a reduction of the $R_{2}^{*}$ in the cortex was found. In the inner medulla increased $R_{2}^{*}$ was found after iodixanol administration. These results showed that BOLD parameters respond to different ionated contrast-media. Donati et al. [42] estimated the order of magnitude of the changes in the renal relaxivity induced by respiratory challenges. The largest BOLD response was measured in the renal medulla during carbogen breathing at $3 \mathrm{~T}$.

In a study performed to evaluate renal function using BOLD MRI in patients affected by chronic kidney disease, Michaely et al. [43] concluded that BOLD MRI is not able to reflect different stages of renal insufficiency. However, as stated by the authors, a main limitation of the study can be seen in the heterogeneous patient population [43].

\section{Functional Contrast-Enhanced MRI}

Technique

In dynamic contrast-enhanced MRI, a gadolinium-chelate (such as Gd-DTPA, gadobutrol, or gadoteric acid) is injected in a typical concentration of $0.1 \mathrm{mmol} / \mathrm{kg}$ body weight as a bolus using a power injector with an injection rate of approximately $2 \mathrm{ml} / \mathrm{s}$. The uptake and the excretion of the contrast-medium are followed using $T_{1}$-weighted sequences applied with relatively high temporal resolution (i.e., in the order of few seconds). Several different techniques of functional contrast-enhanced MRI have been proposed for measuring renal function [44-48]. In the following, we will focus on MR nephrography methods, which allow for assessment of differential renal function and urinary drainage, similar to techniques of nuclear (MAG3 or Tc-DTPA) scintigraphy.

Dynamic nephrography and urography can be carried out with a navigator-gated single-slice TurboFLASH sequence with a non-selective saturation pre-pulse allowing repetitive image acquisition every 4-5 s during continuous breathing with an acquisition time of approximately $0.5 \mathrm{~s}$. Alternatively, standard conventional gradient-echo sequences with high flip angle can be applied. However, the relatively long acquisition time of these sequences hinders breathing compensation. The image plane (typically with $10-30 \mathrm{~mm}$ slice thickness) is slightly angulated from the coronal orientation and positioned according to the longest extension of the kidneys. After the acquisition of a few native images, a gadolinium bolus is injected. $T_{1}$-weighted images are continuously acquired during the 20-30 min that follow bolus injection.

The calculation of the differential renal function is illustrated in Fig. 5. Time-intensity curves are generated from the dynamic data sets regarding regions-of-interest (ROIs) encompassing the renal parenchyma of each kidney. The time-intensity curve of each kidney presents a vascular, parenchymal and excretory phase. After a short vascular phase with a high increase of the signal intensity directly after the administration of the Gd-DTPA bolus, a more prolonged linear increase over 1-2 min can be seen in the parenchymal phase corresponding to the contrast-medium transfer into the glomeruli. The rate of signal intensity increase is directly related to the filtration capacity per tissue volume. In the following excretory phase, the signal intensity decreases again because of the superimposed excretion of the contrast-medium into the collecting system. For calculation of the differential renal function the gradients of the linear increase in the parenchymal phase are measured using a linear least-squares fit and multiplied by the total volume as derived from the selected ROI areas (the latter are taken as a relative estimate for the split renal volume). The calculated product of gradient and volume for one kidney is divided by the sum of the products of both kidneys in order to obtain the split renal function in percent [49].

The described method can be expanded to quantify the global GFR by continuing MR-scanning for altogether 70 min. The idea behind this approach is that most gadoliniumbased contrast agents are exclusively excreted by glomerular filtration and, therefore, can be applied as a filtration marker. After injection, the gadolinium-chelate shows a fast homogeneous distribution into the extracellular space that takes 


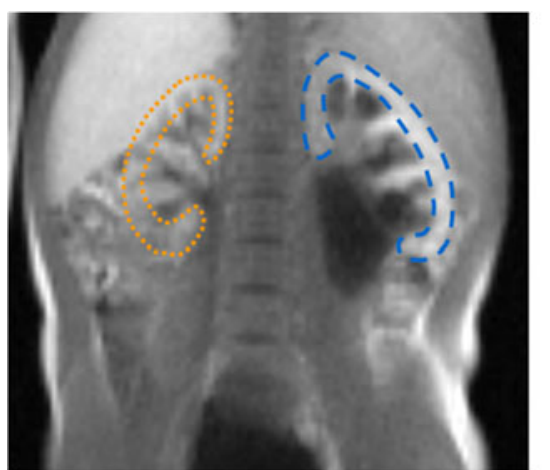

Fig. 5 Calculation of split renal function in a 3-year-old child with ureteropelvic junction stenosis on the left side. ROI definition is indicated to the left. A linear fit is applied to the signal intensities during the parenchymal phase 1-3 min after administration of

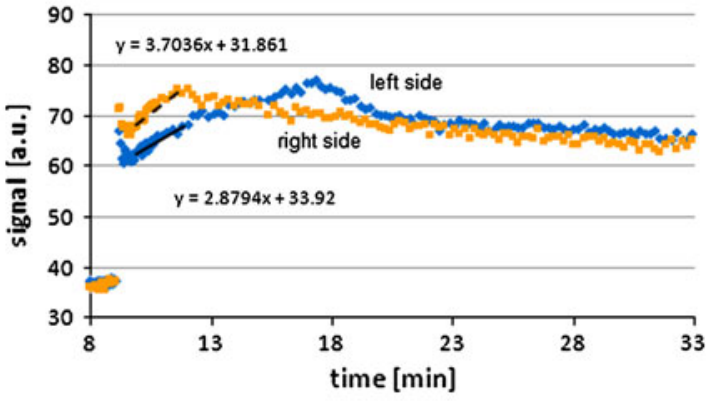

contrast-media. The slope of the signal increase is multiplied with the area of the ROI for one side and divided by the sum of both products in order to obtain the differential renal function (re:li 60:40\%)

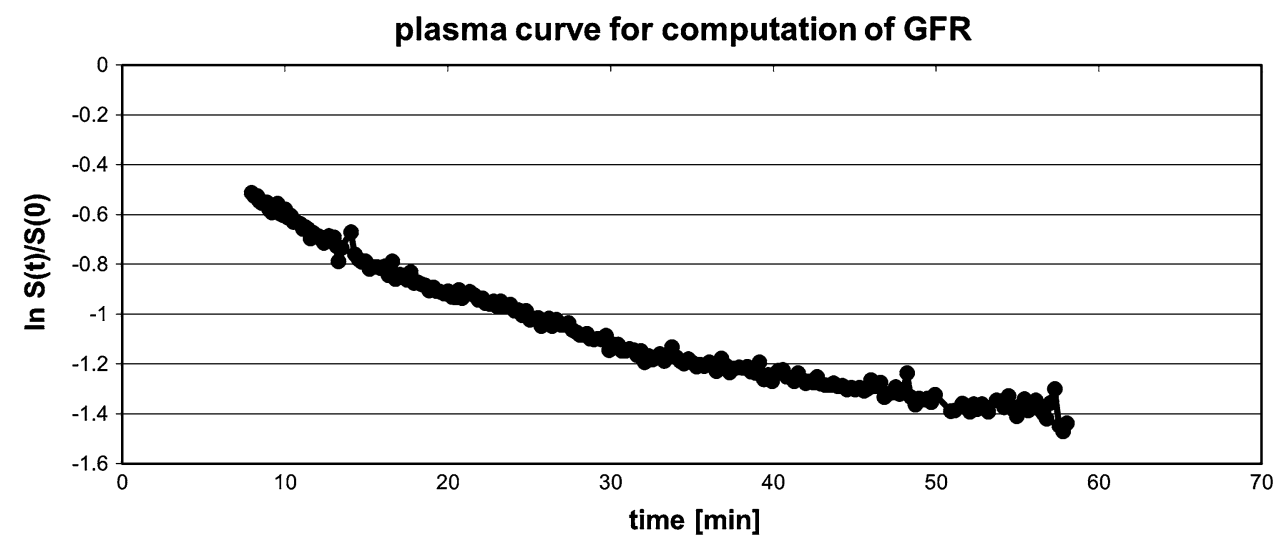

Fig. 6 Monitoring of MR-signal attenuation after administration of gadolinium-containing contrast-media in $T_{1}$-weighted images (saturation-recovery TrueFISP: $\mathrm{TR} / \mathrm{TE}=2.6 / 1.3 \mathrm{~ms}$, flip angle $=70^{\circ}$, recovery time $300 \mathrm{~ms}$ ) allows the quantification of the GFR. GFR is

place in about $20 \mathrm{~min}$. From this time point onwards, the reduction of the tissue concentration is solely determined by glomerular filtration. This signal-intensity decrease due to a reduction in gadolinium-concentration in the extracellular space can now be measured from ROI measurements within the liver [47] (Fig. 6).

\section{Applications}

The above described technique was tested in potential kidney donors [49, 50] with preserved GFR and subsequently expanded to a study evaluating patients with chronic kidney disease with renal filtration reduced down to $30 \mathrm{ml} / \mathrm{min} /$ $1.73 \mathrm{~m}^{2}$. Artunc et al. [50] showed that the GFR can be estimated with reasonable accuracy in a clinical setting. The long measurement time of about $80 \mathrm{~min}$ was reported to constitute a major disadvantage of this approach. However, the measurement of the global GFR can be added to a conventional contrast-enhanced MRI examination without the necessity to apply another injection of contrast agent. evaluated from the renal clearance of gadobutrol within the extracellular fluid volume by exponential fitting of signal-time curves measured over the liver

Compartment analysis for determination of single-sided kidney function is a commonly applied approach. Bokacheva et al. [51] recently compared six frequently applied compartment models. It has been reported that GFR calculation is strongly influenced by the applied model (e.g., two or three compartments) and the definition of the ROI (whole kidney parenchyma or solely renal cortex). A kinetic model with good concordance to the reference method was developed by Vivier et al. [52] for calculation of GFR during a clinical MR investigation of patients with liver cirrhosis. An interesting new approach was reported by Katzberg et al. [53]: GFR is determined from renal blood flow measured with phasecontrast MRI and the renal extraction fraction (REF), which is estimated from the concentration difference as measured by the MRI signal difference between the renal artery and the renal vein. The single-sided GFR is calculated from the product of REF and renal blood flow. Katzberg et al. [53] demonstrated that the described technique provides reasonable accuracy in a cohort of patients with solitary kidneys. 


\section{Conclusion}

Functional MRI has great potential as a non- or minimally invasive tool for accurate assessment of renal tissue viability, early detection of diffuse renal disease, improved characterisation of renal lesions, and safe monitoring of renal function and disease. Several patient collectives may benefit from a multi-modality MR-based diagnostic approach. Firstly, noncontrast-enhanced MRI techniques are potential candidates for follow-up examinations of the kidneys in patients that present a severe renal dysfunction (i.e., GFR $<30 \mathrm{ml} / \mathrm{min} /$ $1.73 \mathrm{~m}^{2}$ ). Secondly, in patients with renal allografts, functional MRI has the potential of providing early stage detection of allograft dysfunction. Finally, contrast-enhanced MR nephrography represents an alternative to nuclear scintigraphy in paediatric patients with anomalies of the urinary tract.

Most of the techniques described in this review aim at the accurate and reproducible quantification of renal biomarkers. Quantitative MRI may allow inter-study comparison (which is especially desirable in the case of multi-centre studies) and, in the best case, support the clinical management of the single patient. However, the dependence of the mentioned biomarkers from study- and populationdependent factors should be further investigated in the future.

In the past years the reproducibility of these functional MRI tools for the assessment of renal function has repeatedly been tested. So far, 1.5 T MR systems have mainly been applied for renal imaging. However, further investigations at higher field strength are necessary to improve SNR and contrast-to-noise ratio of these advanced MRI techniques by maintaining the specific absorption rate within uncritical values and high image quality [54].

Besides the proven feasibility and reproducibility of these techniques, the role of renal functional MRI for clinical management of renal disease should be further investigated.

Disclosures No potential conflicts of interest relevant to this article were reported.

\section{References}

Papers of particular interest, published recently, have been highlighted as:

- Of importance,

-• Of major importance.

1. van Heeswijk RB, Bonanno G, Coppo S, Coristine A, Kober T, Stuber M. Motion compensation strategies in magnetic resonance imaging. Crit Rev Biomed Eng. 2012;40:99-119.

2. - Kim S, Naik M, Sigmund E, Taouli B. Diffusion-weighted MR imaging of the kidneys and the urinary tract. Magn Reson Imaging Clin N Am. 2008;16:585-96. This review article provides a comprehensive overview on the technique of DWI applied to the kidneys and summarizes applications of diffusionweighted MRI for the assessment of diffuse renal diseases and focal renal lesions.

3. Martirosian P, Klose U, Mader I, Schick F. FAIR true-FISP perfusion imaging of the kidneys. Magn Reson Med. 2004;51:353-61.

4. Prasad PV, Edelman RR, Epstein FH. Noninvasive evaluation of intrarenal oxygenation with BOLD MRI. Circulation. 1996;94: 3271-5.

5. Springer F, Martirosian P, Boss A, Claussen CD, Schick F. Current problems and future opportunities of abdominal magnetic resonance imaging at higher field strengths. Top Magn Reson Imaging. 2010;21:141-8.

6. Nehrke K, Bornet P. DREAM-a novel approach for robust, ultrafast, multislice B1 mapping. Magn Reson Med. 2012;68:1517-26.

7. Gauden AJ, Phal PM, Drummond KJ. MRI safety: nephrogenic systemic fibrosis and other risks. J Clin Neurosci. 2010;17:1097-104.

8. Le Bihan D, Breton E, Lallemand D, et al. MR imaging of intravoxel incoherent motions: application to diffusion and perfusion in neurologic disorders. Radiology. 1986;161:401-7.

9. Gaudiano C, Clementi V, Busato F, Corcioni B, Orrei MG, Ferramosca E, Fabbri E, Berardi P, Santoro A, Golfieri R. Diffusion tensor imaging and tractography of the kidneys: assessment of chronic parenchymal diseases. Eur Radiol. 2013; doi: 10.1007/s00330-012-2749-y.

10. Ries M, Jones RA, Basseau F, Moonen CT, Grenier N. Diffusion tensor MRI of the human kidney. J Magn Reson Imaging. 2001;14:42-9.

11. Sigmund EE, Vivier PH, Sui D, Lamparello NA, Tantillo K, Mikheev A, Rusinek H, Babb JS, Storey P, Lee VS, Chandarana $H$. Intravoxel incoherent motion and diffusion-tensor imaging in renal tissue under hydration and furosemide flow challenges. Radiology. 2012;263:758-69.

12. Wittsack HJ, Lanzman RS, Quentin M, Kuhlemann J, Klasen J, Pentang G, Riegger C, Antoch G, Blondin D. Temporally resolved electrocardiogram-triggered diffusion-weighted imaging of the human kidney: correlation between intravoxel incoherent motion parameters and renal blood flow at different time points of the cardiac cycle. Investig Radiol. 2012;47:226-30.

13. Zhang J, Tehrani YM, Wang L, Ishill NM, Schwartz LH, Hricak H. Renal masses: characterization with diffusion-weighted MR imaging - a preliminary experience. Radiology. 2008;247: 458-64.

14. Inci E, Hocaoglu E, Aydin S, Cimilli T. Diffusion-weighted magnetic resonance imaging in evaluation of primary solid and cystic renal masses using the Bosniak classification. Eur J Radiol. 2012;81:815-20.

15. Yu X, Lin M, Ouyang H, Zhou C, Zhang H. Application of ADC measurement in characterization of renal cell carcinomas with different pathological types and grades by 3.0T diffusionweighted MRI. Eur J Radiol. 2012;81:3061-6.

16. Chandarana H, Lee VS, Hecht E, Taouli B, Sigmund EE. Comparison of biexponential and monoexponential model of diffusion weighted imaging in evaluation of renal lesions: preliminary experience. Investig Radiol. 2011;46:285-91.

17. • Thoeny HC, De Keyzer F. Diffusion-weighted MR imaging of native and transplanted kidneys. Radiology. 2011;259:25-38. This review article provides a comprehensive overview on the most recent applications of diffusion-weighted MRI for the assessment of renal allografts dysfunctions.

18. Hueper K, Gutberlet M, Rodt T, Gwinner W, Lehner F, Wacker F, Galanski M, Hartung D. Diffusion tensor imaging and tractography for assessment of renal allograft dysfunction-initial results. Eur Radiol. 2011;21:2427-33.

19. Palmucci S, Mauro LA, Failla G, Foti PV, Milone P, Sinagra N, Zerbo D, Veroux P, Ettorre GC, Veroux M. Magnetic resonance 
with diffusion-weighted imaging in the evaluation of transplanted kidneys: updating results in 35 patients. Transplant Proc. 2012;44:1884-8.

20. Lu L, Sedor JR, Gulani V, et al. Use of diffusion tensor MRI to identify early changes in diabetic nephropathy. Am J Nephrol. 2011;34:476-82.

21. Hueper K, Hartung D, Gutberlet M, Gueler F, Sann H, Husen B, Wacker F, Reiche D. Magnetic resonance diffusion tensor imaging for evaluation of histopathological changes in a rat model of diabetic nephropathy. Investig Radiol. 2012;47:430-7.

22. Boss A, Martirosian P, Graf H, Claussen CD, Schlemmer HP, Schick F. High resolution MR perfusion imaging of the kidneys at 3 Tesla without administration of contrast media. Rofo. 2005; 177:1625-30.

23. Cutajar M, Thomas DL, Banks T, Clark CA, Golay X, Gordon I. Repeatability of renal arterial spin labelling MRI in healthy subjects. MAGMA. 2012;25:145-53.

24. Wu WC, Su MY, Chang CC, Tseng WY, Liu KL. Renal perfusion 3-T MR imaging: a comparative study of arterial spin labeling and dynamic contrast-enhanced techniques. Radiology. 2011;261:845-53.

25. Deibler AR, Pollock JM, Kraft RA, Tan H, Burdette JH, Maldjian JA. Arterial spin-labeling in routine clinical practice, Part 1: technique and artifacts. Am J Neuroradiol. 2008;29:1228-34.

26. Artz NS, Sadowski EA, Wentland AL, Djamali A, Grist TM, Seo S, Fain SB. Reproducibility of renal perfusion MR imaging in native and transplanted kidneys using non-contrast arterial spin labeling. J Magn Reson Imaging. 2011;33:1414-21.

27. Rossi C, Artunc F, Martirosian P, Schlemmer HP, Schick F, Boss A. Histogram analysis of renal arterial spin labeling perfusion data reveals differences between volunteers and patients with mild chronic kidney disease. Investig Radiol. 2012;47:490-6.

28. Pedrosa I, Rafatzand K, Robson P, Wagner AA, Atkins MB, Rofsky NM, Alsop DC. Arterial spin labeling MR imaging for characterisation of renal masses in patients with impaired renal function: initial experience. Eur Radiol. 2012;22:484-92.

29. Lanzman RS, Robson PM, Sun MR, Patel AD, Mentore K, Wagner AA, Genega EM, Rofsky NM, Alsop DC, Pedrosa I. Arterial spin-labeling MR imaging of renal masses: correlation with histopathologic findings. Radiology. 2012;265:799-808.

30. Schor-Bardach R, Alsop DC, Pedrosa I, Solazzo SA, Wang X, Marquis RP, Atkins MB, Regan M, Signoretti S, Lenkinski RE, Goldberg SN. Does arterial spin-labeling MR imaging-measured tumor perfusion correlate with renal cell cancer response to antiangiogenic therapy in a mouse model? Radiology. 2009;251: 731-42.

31. Pedersen M, Dissing TH, Mørkenborg J, Stødkilde-Jørgensen H, Hansen LH, Pedersen LB, Grenier N, Frøkiaer J. Validation of quantitative BOLD MRI measurements in kidney: application to unilateral ureteral obstruction. Kidney Int. 2005;67:2305-12.

32. Simon-Zoula SC, Hofmann L, Giger A, Vogt B, Vock P, Frey FJ, Boesch $\mathrm{C}$. Non-invasive monitoring of renal oxygenation using BOLD-MRI: a reproducibility study. NMR Biomed. 2006;19: 84-9.

33. Xin-Long P, Jing-Xia X, Jian-Yu L, Song W, Xin-Kui T. A preliminary study of blood-oxygen-level-dependent MRI in patients with chronic kidney disease. Magn Reson Imaging. 2012;30:330-5.

34. Yin WJ, Liu F, Li XM, Yang L, Zhao S, Huang ZX, Huang YQ, Liu RB. Noninvasive evaluation of renal oxygenation in diabetic nephropathy by BOLD-MRI. Eur J Radiol. 2012;81:1426-31.

35. - Prasad P, Li LP, Halter S, Cabray J, Ye M, Batlle D. Evaluation of renal hypoxia in diabetic mice by BOLD MRI. Investig Radiol.
2010;45:1-4. This study showed that monitoring of progressive renal hypoxia in a mouse model of diabetic kidney disease is feasible using BOLD MRI.

36. Xiao W, Xu J, Wang Q, Xu Y, Zhang M. Functional evaluation of transplanted kidneys in normal function and acute rejection using BOLD MR imaging. Eur J Radiol. 2012;81:838-45.

37. Park SY, Kim CK, Park BK, Huh W, Kim SJ, Kim B. Evaluation of transplanted kidneys using blood oxygenation level-dependent MRI at $3 \mathrm{~T}$ : a preliminary study. Am J Roentgenol. 2012;198:1108-14.

38. Min JH, Kim CK, Park BK, Kim E, Kim B. Assessment of renal lesions with blood oxygenation level-dependent MRI at $3 \mathrm{~T}$ : preliminary experience. Am J Roentgenol. 2011;197:489-94.

39. Warner L, Glockner JF, Woollard J, Textor SC, Romero JC, Lerman LO. Determinations of renal cortical and medullary oxygenation using blood oxygen level-dependent magnetic resonance imaging and selective diuretics. Investig Radiol. 2011;46:41-7.

40. $\bullet$ Zhang Y, Wang J, Yang X, Wang X, Zhang J, Fang J, Jiang X. The serial effect of iodinated contrast media on renal hemodynamics and oxygenation as evaluated by ASL and BOLD MRI. Contrast Media Mol Imaging. 2012; 7:418-25. This study performed on rabbits showed that the administration of iodinated contrast media results in longer-term hypo-perfusion (assessed using ASL MRI) of the whole kidney and in decreased oxygenation (assessed using BOLD MRI) of the medulla.

41. Haneder S, Augustin J, Jost G, Pietsch H, Lengsfeld P, Krämer BK, Schoenberg SO, Meyer M, Attenberger UI, Michaely HJ. Impact of iso- and low-osmolar iodinated contrast agents on BOLD and diffusion MRI in swine kidneys. Investig Radiol. 2012;47:299-305.

42. Donati OF, Nanz D, Serra AL, Boss A. Quantitative BOLD response of the renal medulla to hyperoxic challenge at $1.5 \mathrm{~T}$ and 3.0 T. NMR Biomed. 2012;25:1133-8.

43. Michaely HJ, Metzger L, Haneder S, Hansmann J, Schoenberg SO, Attenberger UI. Renal BOLD-MRI does not reflect renal function in chronic kidney disease. Kidney Int. 2012;81:684-9.

44. Lee VS, Rusinek H, Noz ME, Lee P, Raghavan M, Kramer EL. Dynamic three-dimensional MR renography for the measurement of single kidney function: initial experience. Radiology. 2003;227: 289-94.

45. Hackstein N, Heckrodt J, Rau WS. Measurement of single-kidney glomerular filtration rate using a contrast-enhanced dynamic gradient-echo sequence and the Rutland-Patlak plot technique. J Magn Reson Imaging. 2003;18:714-25.

46. Buckley DL, Shurrab AE, Cheung CM, Jones AP, Mamtora H, Kalra PA. Measurement of single kidney function using dynamic contrast-enhanced MRI: comparison of two models in human subjects. J Magn Reson Imaging. 2006;24:1117-23.

47. Boss A, Martirosian P, Gehrmann M, Artunc F, Risler T, Oesingmann N, Claussen CD, Schick F, Küper K, Schlemmer HP. Quantitative assessment of glomerular filtration rate with MR gadolinium slope clearance measurements: a phase I trial. Radiology. 2007;242:783-90.

48. Rohrschneider WK, Haufe S, Wiesel M, Tönshoff B, Wunsch R, Darge K, Clorius JH, Tröger J. Functional and morphologic evaluation of congenital urinary tract dilatation by using combined static-dynamic MR urography: findings in kidneys with a single collecting system. Radiology. 2002;224:683-94.

49. Rossi C, Boss A, Artunc F, Yildiz S, Martirosian P, Dittmann H, Claussen CD, Heyne N, Schick F, Schlemmer HP. Comprehensive assessment of renal function and vessel morphology in potential living kidney donors: an MRI-based approach. Investig Radiol. 2009;44:705-11. 
50. Artunc F, Yildiz S, Rossi C, Boss A, Dittmann H, Schlemmer HP, Risler T, Heyne N. Simultaneous evaluation of renal morphology and function in live kidney donors using dynamic magnetic resonance imaging. Nephrol Dial Transplant. 2010;25:1986-91.

51. Bokacheva L, Rusinek H, Zhang JL, Chen Q, Lee VS. Estimates of glomerular filtration rate from MR renography and tracer kinetic models. J Magn Reson Imaging. 2009;29:371-82.

52. Vivier PH, Storey P, Rusinek H, Zhang JL, Yamamoto A, Tantillo K, Khan U, Lim RP, Babb JS, John D, Teperman LW, Chandarana H, Friedman K, Benstein JA, Skolnik EY, Lee VS. Kidney function: glomerular filtration rate measurement with MR renography in patients with cirrhosis. Radiology. 2011; 259:462-70.

53. Katzberg RW, Buonocore MH, Low R, Hu B, Jain K, Castillo M, Troxel S, Nguyen MM. MR determination of glomerular filtration rate in subjects with solitary kidneys in comparison to clinical standards of renal function: feasibility and preliminary report. Contrast Media Mol Imaging. 2009;4:51-65.

54. Rosenkrantz AB, Oei M, Babb JS, Niver BE, Taouli B. Diffusionweighted imaging of the abdomen at 3.0 Tesla: image quality and apparent diffusion coefficient reproducibility compared with 1.5 Tesla. J Magn Reson Imaging. 2011;33:128-35. 